\title{
OBSERVATIONS OF INFRARED EMISSION FROM GALAXIES
}

\author{
P. F. ROCHE \\ Royal Observatory Edinburgh
}

\begin{abstract}
Aspects of the infrared emission from galaxies obtained from groundbased observations are described. There are clear differences between the dust in galaxies with active nuclei and those dominated by nuclear HII regions. In particular the family of unidentified emission bands between 3 and $13 \mu \mathrm{m}$ dominate the spectra in the latter objects, but are rarely evident in spectra of active nuclei. However results from spatial, spectral and polarization measurements show that many of the dust properties in galaxies are broadly similar to those in the Milky Way.
\end{abstract}

\section{INTRODUCTION}

For the purposes of discussing the infrared emission from galaxies, it is useful to distinguish the different types of galaxies according to the nature of the dominant emission; as this is reflected in the infrared properties. I will discuss, briefly, the emission from active galaxy nuclei (AGN) where the fundamental energy source is believed to be derived from a non-thermal mechanism, and probably accretion of material by a massive nuclear black hole. I will then concentrate on the socalled 'starburst' galaxies, where the nuclear regions are dominated by vigorous star formation. The quiescent and most elliptical galaxies usually have IR emission that is too weak for detailed study with present instruments. It is important to bear in mind that all galaxies that have been investigated in detail are bright in the infrared, and so we are dealing with what is effectively an infrared-selected sample. Because of the limited space available here, I will concentrate on a few examples of the ways that the infrared emission from galaxies can be probed. More wide-ranging reviews on dust in galaxies can be found in Joseph (1988) and Lonsdale (1987) and on continuum emission from AGN in Gear (1988).

Unlike Galactic objects, where it is often possible to isolate a particular target for detailed study, observations of galaxies usually encompass a relatively large volume and can include a wide range of different phenomena. Typical aperture sizes employed range from $<1 \mathrm{pc}$ in the Magellanic Clouds to $>1 \mathrm{kpc}$ in distant objects. By comparison, Galactic objects are usually studied with beams that are fractions of a pc, so that diffuse emission is likely to be correspondingly more important in observations of galaxies. 
Although non-thermal emission processes can be important in luminous AGN, and starlight, emission from circumstellar shells and ionic and molecular line emission can all contribute in many galaxies, it is emission from interstellar dust that provides by far the greatest part of the infrared output in most galaxies. Apart from the study of extragalactic dust for its own intrinsic interest, where galaxies provide a much greater range of excitation and abundance variations than is found in the Milky Way, there are several other reasons why this is important. Only a relatively small amount of dust is required to absorb a substantial fraction of the optical and ultraviolet light emitted by a galaxy, so an understanding of the dust emission and absorption properties is vital to unravelling the energetics of galaxies; indeed in some cases, the dust absorption towards galaxy nuclei can lead to visible extinctions of many tens of magnitudes, so that only studies at infrared or radio wavelengths can probe these regions. Further, extinction may be one of the key differences between Seyferts of type I and II (see Lawrence, 1987).

\section{SPATIAL STUDIES}

Imaging and mapping in the infrared can reveal the spatial and temperature distributions of the emitting materials. AGN are sufficiently compact (with the so-called broad-line region perhaps a pc or so, and the narrow-line region a couple of hundred parsecs in diameter), that it is difficult to resolve spatial structure in the infrared, although the array detectors that are now available render this possible for relatively nearby examples, e.g. Tresch-Feinberg et al. (1987) have found structure in the nucleus of NGC 1068 on a scale of about an arcsec. However, other approaches are possible. Clavel et al. (1988), using variability studies, have found that the 3 $\mu \mathrm{m}$ light curve of the Seyfert 1 galaxy, Fairall 9, lags behind that of the UV and optical continuum, and infer that hot dust, emitting at a temperature of $\sim 1000$ $K$, lies about 400 light days from the nuclear source, commensurate with the values expected for heating of grains by the nucleus. Galaxies with powerful nuclear HII regions are more extended, with typical sizes of hundreds to a couple of thousand pc, and so are more amenable to spatial studies. In general, there is a good correspondence between the spatial distribution of infrared emission and that of the radio, blue light, or $\mathrm{H} \alpha$ emission, although there are often differences in detail (e. $g$. Telesco \& Gatley, 1984; Telesco et al., 1987), in particular, the effects of extinction can distort considerably the view obtained in the visible. In these galaxies, the brightest regions at mid- and far-infrared wavelengths are those that show evidence of intense recent star-formation, where dust is heated by young, hot stars. Because of limited instrumental sensitivity, mid-IR observations are usually confined to the central regions, but these often contain most of the galaxies output at these wavelengths. In the far-infrared, emission from cool dust in the outer galaxy disks may be important (Smith, 1982; Thronson et al., 1987a).

\section{SPECTRAL PROPERTIES}

The spectral energy distribution obviously contains a great deal of information on the temperature of the emitting dust, and at long wavelengths, where the emission is in the Rayleigh limit, it can give fairly good estimates of the total mass of cool dust. The cool dust is best studied at sub-mm wavelengths and, now that large dedicated sub-mm dishes are operating, reliable estimates of the temperature, mass 
and distribution of the coldest dust should become available in the near future. Initial results have been published by Telesco \& Harper (1980), Thronson et al. (1987b) and Chini et al. (1986). The IRAS data cover the region around the peak of the IR emission which, in most objects lies in the far-infrared (Helou, 1989). At shorter wavelengths, the emission from hot dust dominates, but the effects of extinction can be important, and in AGN it is possible that non-thermal emission may be present.

The overall energy distributions of galaxies containing powerful AGN are often very different from those dominated by nuclear HII regions, in that they are much flatter, and often have peaks in the mid-infrared, presumably due to emission from warm dust heated by the active nuclei. The most luminous objects have spectra that may be approximated by a power law function throughout the infrared, although radio-quiet objects must turn over somewhere in the sub-mm region. In these objects which appear to have smoothly varying spectral distributions, it is difficult to determine what the contributions from dust or non-thermal emission are; measurements of high photometric accuracy, studies of variability (e.g. Robson et al., 1986; Gear et al., 1985) or spectroscopy may reveal details that can disentangle the various components.

Infrared spectroscopy can probe the amount of extinction present, not only from the observed ratios of hydrogen Brackett and Paschen lines, but also from the shape of the continuum and the depth of any silicate feature at $10 \mu \mathrm{m}$. At the same time, the presence of emission features can give information on the nature of the dust grains and allow comparison with Galactic sources. Spectroscopy is now available for a large number of galaxy nuclei in the 8-13 $\mu \mathrm{m}$ region (Aitken \& Roche, 1985; Roche \& Aitken, 1985, and in preparation), in the 3-3.5 $\mu \mathrm{m}$ region (Moorwood, 1986), and for a few objects at $20 \mu \mathrm{m}$ (Houck et al., 1980) and near infrared wavelengths (Cutri, et al., 1981; Joseph, 1987).

The most striking result from these studies is that the galaxies with powerful non-thermal nuclei have very different spectral properties from those dominated by nuclear HII regions. The smooth power-law emission seen in the broad-band energy distributions of luminous AGN continues to be evident when they are examined at higher spectral resolution, where objects such as the quasar $3 \mathrm{C} 273$ or the type-I Seyfert NGC 4151 have smooth featureless spectra in the near-IR and $10 \mu \mathrm{m}$ regions (Lee et al., 1982; Roche et al., 1984; Aitken \& Roche, 1985). Of course, this does not mean that there is no warm dust emitting in these regions, but it does mean that if dust is responsible for a substantial fraction of the emission, it must be devoid of strong emission features at these wavelengths. Indeed, a weak emission peak near $3 \mu \mathrm{m}$ which may be due to emission from hot dust is often present in luminous AGN (e.g. Edelson \& Malkan, 1986). Less luminous AGN show more evidence of spectral structure, with a substantial fraction having minima near 10 $\mu \mathrm{m}$ attributable to silicate grains; silicate emission may be present in some objects (Aitken \& Roche, 1985).

By contrast, HII region galaxies show a great deal of spectral structure. About $90 \%$ of those that have been measured spectroscopically at $10 \mu \mathrm{m}$ are dominated by the strong emission peaks near $11.3,8.7$ and $7.7 \mu \mathrm{m}$, which are part of the family of unidentified infrared bands (UIR) discussed at length in these proceedings. This is in almost complete contradistinction to the AGN where the UIR bands are detected only rarely and, where they are seen are apparently associated with circumnuclear star-formation regions (Cutri et al., 1984; Roche, 1987). Similar results have been 
obtained by Moorwood (1986) who found that galaxies with evidence of nuclear HII regions have strong emission in the $3.3 \mu \mathrm{m}$ UIR band, while this feature is absent in AGN. The fraction of starburst galaxies whose spectra are dominated by the UIR emission is considerably higher than in the various classes of Galactic objects in which these emission features are present. This implies that the excitation and/or production of the particles that produce the UIR bands is very effective in starburst nuclei. By analogy with Galactic sources such as the Orion ionization front (Aitken et al., 1979) or NGC 7027 (Aitken \& Roche, 1983), it is likely that the emission features arise just outside the ionized zones of HII regions, and the high flux of non-ionizing photons escaping from the nuclear HII regions and bathing the central region of the starburst galaxies will then be very efficient in exciting this emission.
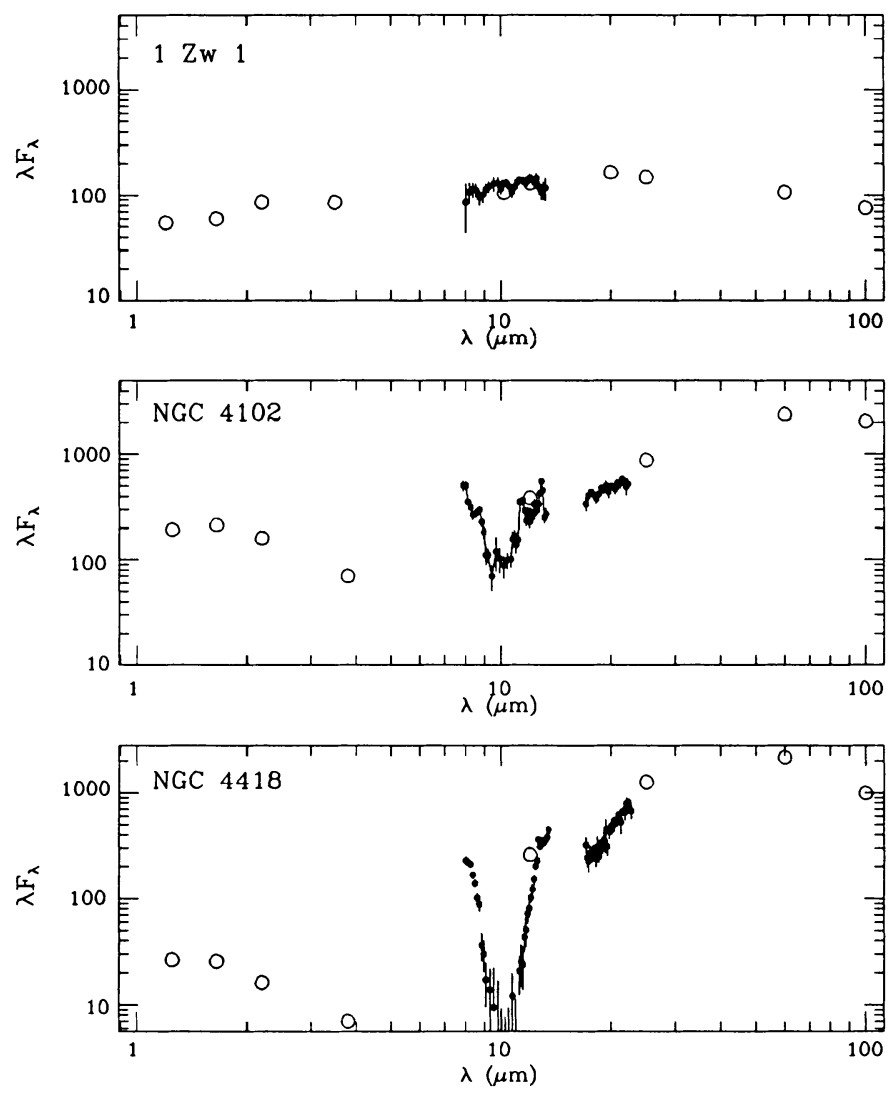

Fig. 1. Spectral energy distributions of 3 galaxies. $1 \mathrm{Zw} 1$ is a Seyfert nucleus with a spectrum that approximates to a power law in the infrared, but with evidence of weak emission peaks near 3 and $20 \mu \mathrm{m}$. NGC 4102 is a starburst nucleus whose mid-infrared spectrum is dominated by the UIR dust emission features, whilst although the $10 \mu \mathrm{m}$ minimum in NGC 4418 is qualitatively quite similar, it is produced by absorption by a large column of cool silicate grains. 
The presence of strong UIR band emission makes it difficult to measure any spectral structure due to silicate grains. The strong 7.7 and $8.7 \mu \mathrm{m}$ bands combine with the $11.3 \mu \mathrm{m}$ feature and long wavelength continuum to form a minimum near $10 \mu \mathrm{m}$ which can mimic the silicate absorption dip. Estimates of any contribution to the spectrum from silicate absorption can be made from extinction measurements derived from the near-IR Brackett lines, or by assuming a canonical starburst galaxy nuclear spectrum and estimating any extra extinction at $10 \mu \mathrm{m}$ from the apparent depth of the minimum compared to the strength of the emission features (Aitken \& Roche, 1984). These approaches are fairly uncertain, but it does seem that at least some starburst nuclei do have a significant component of silicate absorption in their $10 \mu \mathrm{m}$ spectra. The absence of the UIR bands in AGN may result from destruction of the band carriers in the harsh environment around the active nucleus, although the dominance of a single energetic source of luminosity, rather than the extended HII regions in a starburst nucleus may also be important.

The presence of silicate absorption is, however, very evident in the spectra of some galaxies; where the optical depth at $10 \mu \mathrm{m}$ is low, this is most readily seen in objects where the UIR emission is relatively weak. Galaxies such as NGC 4418 (Roche et al., 1986) or NGC 7479 (data in preparation) show substantial silicate optical depths corresponding, in the former case, to $A_{V}>50 \mathrm{mag}$ and rendering the nucleus invisible even at near infrared wavelengths. In these objects there are substantial columns of dust along the line of sight to the nuclei and an understanding of the nature of the object can only be gleaned with knowledge of the dust properties.

Examples of the different galaxy energy distributions are shown in Fig 1.

\section{POLARIZATION}

Another technique that can be employed to probe the effects and nature of dust is the study of polarization. As with observations made to investigate the extinction curve, polarization measurements in the visible and near-infrared regions are hampered because it is difficult to ensure freedom from optical depth effects when observing a complex region such as part of a galaxy. Opportunities occasionally arise where these difficulties are minimised, and the observations of the supernova that exploded in the dust lane of Cen A, SN 1986G, provides such a case where the measurements of the polarization produced by grains in Cen $\mathbf{A}$ could be directed towards a well defined target. Broad band polarimetry from 0.4 to $2 \mu \mathrm{m}$ showed that the polarization data could be fit by the Serkowski curve, and with $\mathrm{P}_{\max }=0.43$ $\mu \mathrm{m}$, implies that the aligned grains are somewhat smaller on average than typical in the Galaxy (Hough et al., 1987). Polarization measurements between 0.4 and 13 $\mu \mathrm{m}$ have been used by Bailey et al. (1988) to isolate three polarization components which they attribute to optical light scattered from a Seyfert 1 nucleus, a near-IR peak which may be a direct view of the nucleus polarized by absorption by aligned grains and mid-IR polarization, orthogonal to that in the near-IR, due to thermal emission from warm aligned grains; because there is no evidence of a polarization peak near $10 \mu \mathrm{m}$, the emitting grains cannot be dominated by silicates.

\section{CONCLUSIONS}

1. Dust in extragalactic objects is broadly similar to that in the Milky Way. Observations of the polarization curve in Cen $\mathbf{A}$ show that it has a similar 
wavelength dependence to the Galaxy, although there appear to be real differences in grain size. Many of the infrared spectral features seen in Galactic objects have been measured in galaxy nuclei; the presence of silicate grains can be inferred in some cases and the particles that give rise to the UIR emission bands are excited efficiently in starburst galaxies. As yet, there are insufficient data to investigate any dependence of grain properties with element abundances; objects such as blue compact galaxies and the Magellanic clouds are obvious targets.

2. The emission from active galactic nuclei is quite different from that from galaxies with nuclear HII regions. The vast majority of the latter have 3-13 $\mu \mathrm{m}$ spectra dominated by the UIR emission bands, but most AGN show only weak spectral structure with some objects showing evidence of silicate grains; this may indicate destruction of the UIR band carriers by the active nucleus.

3. The UIR emission is virtually ubiquitous in starburst galaxy nuclei, and probably arises from the excitation of small grains by the intense flux of non-ionising photons flooding out from the nuclear HII regions. The ratio of 12 to $100 \mu \mathrm{m}$ emission in these galaxies is similar to that found in normal spirals, suggesting that the emission is strongly related to the IR 'cirrus' seen in the Galaxy.

4. About $1 \%$ of the total luminosity of the starburst galaxies is emitted in the UIR features and, as at least some of the continuum emission in the near and mid-infrared is probably emitted by the same particles that give rise to the emission features, it appears that non-equilibrium emission from these particles accounts for several percent of the total luminosity of these galaxies.

5. Silicate absorption is clearly seen in some very heavily obscured galaxies and may be present in many others, where the strong UIR bands render it difficult to separate emission and absorption features.

\section{REFERENCES}

Aitken, D. K. \& Roche, P. F. 1983, M. N. R. A. S., 202, 1233.

. 1984, M. N.R.A.S., 208, 751 .

. 1985, M. N. R. A.S., 213, 777.

Aitken, D. K., Roche, P. F., Spenser, P. M. \& Jones, B. 1979, Astr. Ap., 76, 60.

Bailey, J., Axon, D. J., Hough, J. H., Ward, M. J., McLean, I. \& Heathcote, S. R. 1988, M. N. $R$. $A$. $S$., in press.

Chini, R, Kreysa, E., Krugel, E. \& Mezger, P. G. 1986, Astr. Ap., 166, L8.

Clavel, J., Wamsteker, W. \& Glass, I. S. 1988, preprint.

Cutri, R. M., et al. 1981, Ap. J., 245, 818.

Cutri, R. M., Rudy, R. J., Rieke, G. H., Tokunaga, A. T. \& Willner, S. P. 1984, Ap. J., $280,521$. item Edelson, R. A. \& Malkan, M. A. 1986, Ap. J., 308, 59.

Gear, W. K. 1988, in Millimetre and Submillimetre Astronomy, (Dordrecht: Kluwer), p. 307.

Gear, W. K., Gee, G., Robson, E. I. \& Nolt, I. G. 1985, M. N. R. A. S., 217, 281.

Helou, G. 1989, in IAU Symposium 195, Interstellar Dust, eds. L. J. Allamandola and A. G. G. M. Tielens, (Dordrecht: Kluwer), p. 285.

Houck, J. R., Forrest, W. J. \& McCarthy, J. F. 1980, Ap. J., 242, L65.

Hough, J. H., Bailey, J. A., Rouse, M. F. \& Whittet, D. C. B. 1987, M. N. R. A. S., 227, 1 P.

Lonsdale, C. J. 1987, in Star Formation in Galaxies, (Cal Tech).

Joseph, R. D. 1988, in Dust in the University, (C.U.P.), in press.

Joseph, R. D., Wright, G. S., Wade, R., Graham, J. R., Gatley, I. \& Prestwich, A. H. 1987, in Star Formation in Galaxies, p. 421. 
Lawrence, A. 1987, Pub. A. S. P., 99, 309.

Lee, T. J., Beattie, D. H., Gatley, I., Brand, P. W. J. L., Jones, T. \& Hyland, A. R. 1982, Nature, $295,214$.

Londsdale, C. J. 1987, in Star Formation in Galaxies, (Cal Tech).

Moorwood, A. F. M. 1986, Astr. Ap., 166, 4.

Robson, E. I., Gear, W. K., Brown, L. M. J., Courvoisier, T. J-L., Smith, M. G., Griffin, M. J. \& Blecha, A. 1986, Nature, $\mathbf{3 2 3}, 134$.

Roche, P. F. 1987, in Polycyclic Aromatic Hydrocarbons and Astrophysics, (Dordrecht: Reidel), p. 307.

Roche, P. F., Aitken, D. K., Phillips, M. M. \& Whitmore, B. 1984, M. N. R. A. S., $207,35$.

Roche, P. F. \& Aitken, D. K. 1985, M. N. R. A. S., 213, 789.

Roche, P. F., Aitken, D. K., Smith, C. H. \& James, S. D. 1986, M. N. R. A. S., 218, 19 P.

Smith, J. 1982, Ap. J., 261, 463.

Telesco, C. M. \& Gatley, I. 1984, Ap. J., 284, 557.

Telesco, C. M. \& Harper, D. A. 1980, Ap. J., 235, 392.

Telesco, C. M., Decher, R., Ramsay, B. D., Wolstencroft, R. W. \& Leggett, S. K. 1987, in Star Formation in Galaxies, p. 497.

Thronson, H. A., Walker, C. K., Walker, C. E. \& Maloney, P. 1987b, Ap. J., \$18, 645.

Thronson, H. A., Hunter, D. A., Telesco, C. M., Harper, D. A. \& Decher, R. 1987b, Ap. J., 817, 180.

Tresch-Fienberg, R., Fazio, G. G., Gezari, D. Y., Hoffmann, W. F., Lamb, G. M., Shu, P. K. \& McCreight, C. R. 1987, Ap. J., 312, 542. 


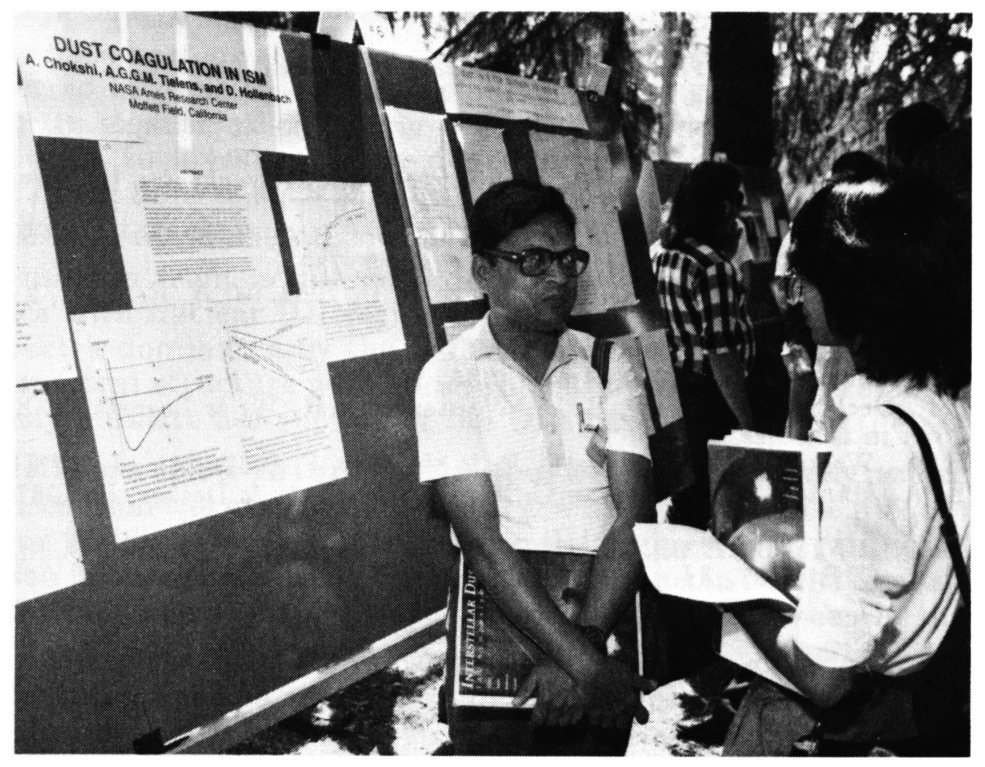

S. K. Jain and Arati Chokshi 